\title{
The impacts of COVID-19 on glaucoma patient outcomes as assessed by POEM
}

\author{
Rathin Pujari ${ }^{1}$ - Geoffrey Chan ${ }^{1,2} \cdot$ Ian Tapply $^{1} \cdot$ Addenbrookes Glaucoma COVID response consortium $^{1}$. \\ Rupert R. Bourne ${ }^{1,3}$
}

Received: 9 December 2020 / Revised: 11 December 2020 / Accepted: 19 January 2021 / Published online: 9 February 2021

(c) Crown 2021

\section{To the Editor:}

We look on with concern, as the COVID-19 virus pandemic continues to disrupt the delivery of ophthalmic care. Whilst the focus has remained on deaths due to COVID-19, the indirect effects related to reductions in the provision of ophthalmic services should not be underestimated. Emerging data has also shown increased patient anxiety and concern regarding their eye care.

The patient-reported outcome and experience measure (POEM) is a validated eight-item questionnaire for use in glaucoma [1,2]. The questionnaire addresses aspects of outcome (acceptability of treatment, fear of blindness, impact on daily life) and aspects of

Presentation of this work and link to additional material: While conducting this research, we also used conversations with four patients to brief an actress who prepared a monologue that highlights these key areas, accessible via the following link, https://player.vimeo.com/ video/488126493. This was screened in an online seminar entitled 'A 360 degree look at new care models in glaucoma-making virtual clinics work for patients, clinicians and service providers' on 15th October 2020, organised and funded by Santen UK Limited.

Members of the Addenbrookes Glaucoma COVID response consortium are listed above References.

Supplementary information The online version contains supplementary material available at https://doi.org/10.1038/s41433021-01425-0.

$\triangle$ Rupert R. Bourne

Rupert.bourne@addenbrookes.nhs.uk

1 Cambridge Eye Research Centre, Department of Ophthalmology, Addenbrooke's Hospital, Cambridge University Hospital NHS,

Cambridge, UK

2 Centre for Ophthalmology and Visual Science, The University of Western Australia, Perth, WA, Australia

3 School of Medicine, Vision and Eye Research Institute, Anglia Ruskin University, Cambridge, UK experience (safety, understanding, organization of care). We used POEM to investigate the change in glaucoma patient experience and attitudes caused by the COVID pandemic.

One hundred and twenty-six consecutive patients attending a tertiary centre glaucoma clinic over a 2-week period in September 2020 were surveyed. An additional column explicitly asking if each POEM item in question was more of a concern during the COVID pandemic was added to the questionnaire (Appendix 1). Questionnaire responses conducted during the pandemic were compared to those pre-pandemic. The questionnaires were conducted anonymously with no pairing to clinical data or demographics.

Survey results showed that the largest change in terms of percentage of patients who agree or strongly agree occurred in item Q2, Q4, Q7 and Q8, which all showed at least a $10-15 \%$ decrease in agreement from pre COVID questionnaire (Fig. 1). Patients identified Q6, Q7 and Q8 to be the aspects that COVID has affected most with $28.8 \%, 32.1 \%$ and $40.2 \%$ 'yes' responses, respectively (Fig. 2).

POEM items Q7 and Q8 pertains to patients' perception of their treatment team, whilst Q2 represents the way glaucoma is treated. These large percentage changes show increasing uncertainty amongst patients about how their glaucoma is managed during the crisis. Impaired confidence in the glaucoma team may not only cause unnecessary concern but cause issues with compliance that could contribute to disease progression.

Responses to Q4 demonstrate that patients are increasingly less confident that the progression of their glaucoma remains stable. In addition, we found that the cohort of patients most concerned about losing vision is $25 \%$ more likely to tick 'yes' when asked if COVID had caused more concern, indicating that the COVID crisis has explicitly exacerbated these concerns. This is especially worrying as even before the crisis, glaucoma units were already 
A. Pre-COVID-19 POEM Responses
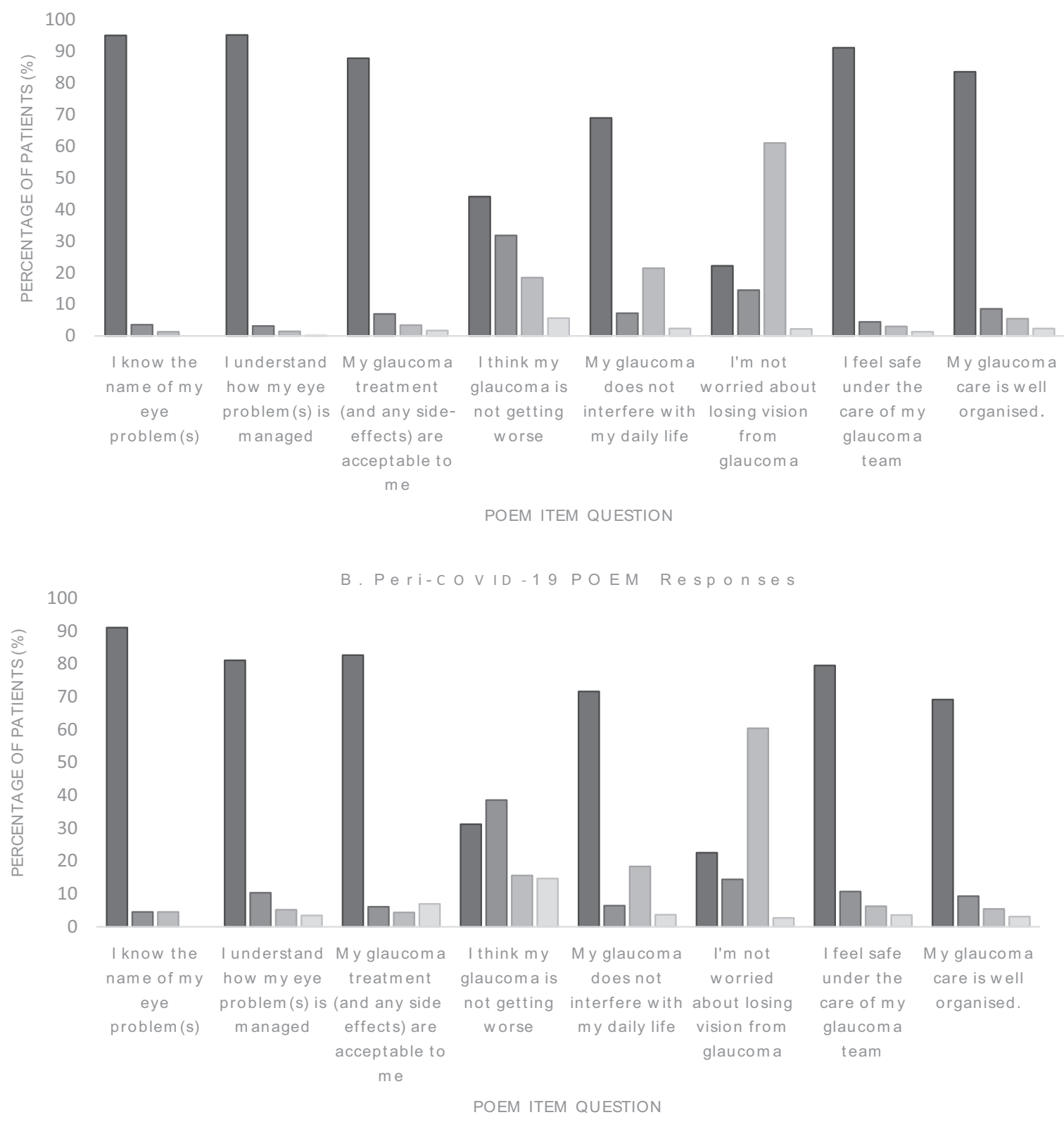

$\square$ Strongly Agree/Agree

$\square$ Not sure

Fig. 1 Comparison of pre-COVID patient report outcomes to those in the peri-COVID era using the POEM Item Questionnaire. Comparison of the two graphs (A: pre-COVID, B: post-COVID) reveals that the largest decrease (at least 10\%) in 'strongly agree

struggling with a backlog of patients, with some delays resulting in loss of vision [3].

Overall, the results of our study show that patients are more uncertain of their glaucoma care. This may reflect the heightened anxiety associated with COVID as well as generalized delays in clinic follow up during the COVID pandemic. Pre-pandemic, many glaucoma units were already managing a backlog of outpatients, associated

\section{- Strongly Disagree/Disagree $\square$ Unable to rate}

responses' occurs in Q2 (patient's understanding of their treatment for glaucoma), Q4 (progression of glaucoma), Q7 and Q8 (patient's perception and confidence in their treatment team).

with heightened anxiety around aspects of glaucoma care [4]. The POEM questionnaire highlights that this situation has been exacerbated by COVID. Further studies investigating delays in patient appointments with impacts on visual acuity deterioration as well as true glaucomatous progression will further elucidate the psychological and physiological impacts that COVID has had. 
Fig. 2 Patient responses identifying if COVID had caused increased concern based on each item of the POEM Questionnaire.

Domains pertaining to safety (Q7) and organizational aspects (Q8) of glaucoma treatment (both related to the perception by the patient of the treatment team) were the areas which patients explicitly identified as areas of concern due to COVID.

This correlated with the results from graphs in Fig. 1 when comparing pre-COVID and post-COVID POEM results.

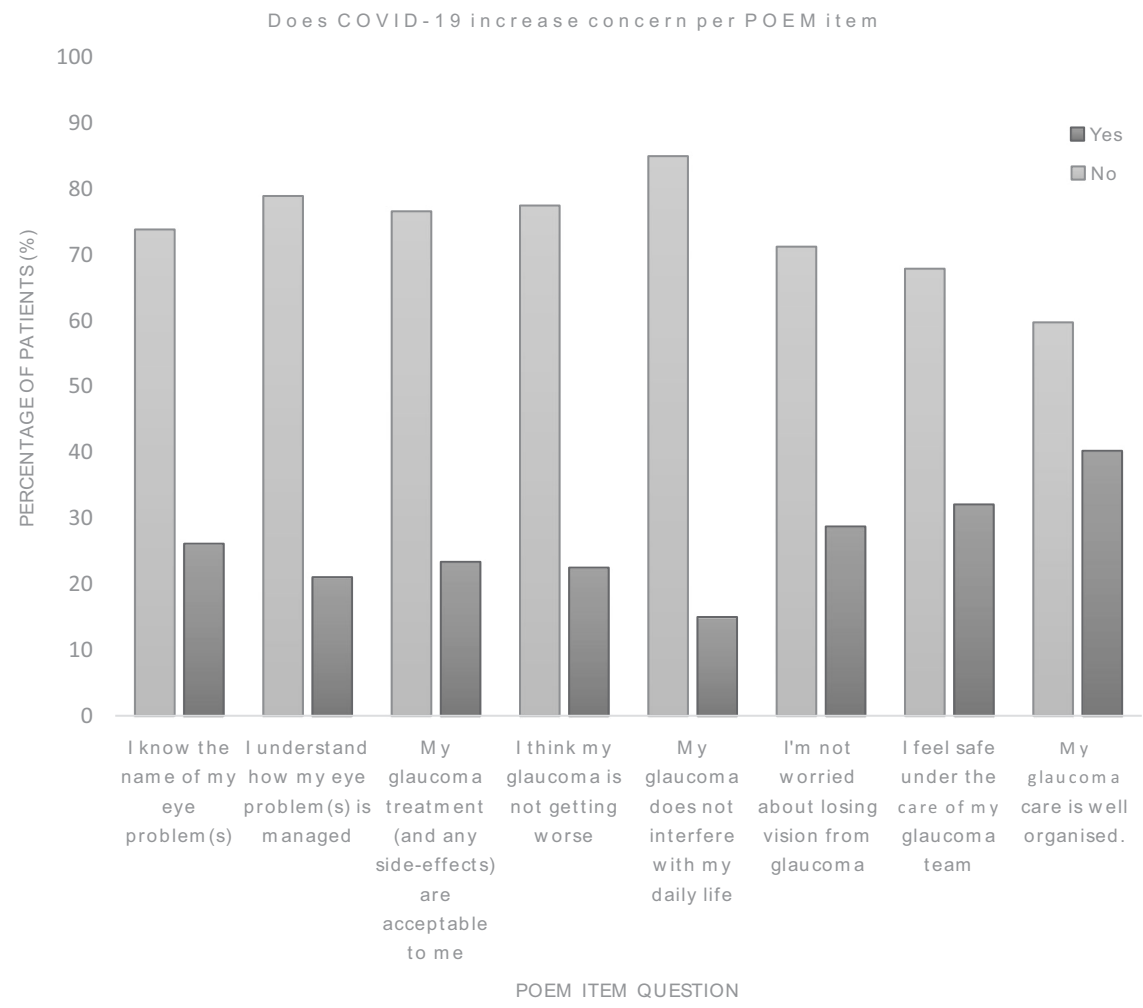

Addenbrookes Glaucoma COVID response consortium Maaz A. Khan ${ }^{1}$, Bryan L. C. Loh ${ }^{1}$, Gopija Nanthagopan ${ }^{1}$, Arun James Thirunavukarasu ${ }^{1}$, Aaron P. L. Fleming ${ }^{1}$, Suraya Yusuf ${ }^{1}$, Xiu Sheng Tan ${ }^{1}$, Shravan V. Gowrishankar ${ }^{1}$, Mrudula Utukuri ${ }^{1}$, Gabriella C. A. Gilks ${ }^{1}$, Marie-Julie Anna Maria Cnudde ${ }^{1}$, Sajan Rajani ${ }^{1}$, Lionel Dufour ${ }^{1}$, Reema Pujari ${ }^{1}$, Rynda Nitiahpapand $^{1}$, Paolo Scollo ${ }^{1}$, Rupert Bourne ${ }^{1}$, Geoffrey Chan ${ }^{1}$, Sharon Springdal ${ }^{1}$, Daniel Kelly ${ }^{1}$, Amy Page ${ }^{1}$

\section{Compliance with ethical standards}

Conflict of interest The authors declare that they have no conflict of interest.

Publisher's note Springer Nature remains neutral with regard to jurisdictional claims in published maps and institutional affiliations.

\section{References}

1. Somner JEA, Sii F, Bourne RR, Cross V, Burr JM, Shah P. Moving from PROMs to POEMs for glaucoma care: a qualitative scoping exercise. Investig Ophthalmol Vis Sci. 2012;53:5940-7.

2. Fraenkel A, Lee GA, Vincent SJ, Vincent RA, Bourne RRA, Shah P. Lessons learned from the development and implementation of a patient-reported outcome and experience measure (POEM) in an Australian glaucoma practice. BMC Ophthalmol. 2019;19:192.

3. Foot B, MacEwen C. Surveillance of sight loss due to delay in ophthalmic treatment or review: frequency, cause and outcome. Eye. 2017;31:771-5.

4. Jayaram H, Strouthidis NG, Gazzard G. The COVID-19 pandemic will redefine the future delivery of glaucoma care. Eye. 2020;34: 1203-5. 